\title{
THE
}

UNIVERSITY

University of Rhode Island

OF RHODE ISLAND

DigitalCommons@URI

$11-13-2009$

\section{The Thermodynamic and Ground State Properties of the TIP4P Water Octamer}

E. Asare

A-R. Musah

E. Curotto

David L. Freeman

University of Rhode Island, dfreeman@uri.edu

J. D. Doll

Follow this and additional works at: https://digitalcommons.uri.edu/chm_facpubs

Terms of Use

All rights reserved under copyright.

\section{Citation/Publisher Attribution}

Asare, E., Musah, A.-R., Curotto, E., Freeman, D. L., \& Doll, J. D. (2009). The Thermodynamic and Ground State Properties of the T1P4P Water Octamer. Journal of Chemical Physics, 131(18), 184508. doi: 10.1063/1.3259047

Available at: http://dx.doi.org/10.1063/1.3259047

This Article is brought to you for free and open access by the Chemistry at DigitalCommons@URI. It has been accepted for inclusion in Chemistry Faculty Publications by an authorized administrator of DigitalCommons@URI. For more information, please contact digitalcommons-group@uri.edu. 


\title{
The thermodynamic and ground state properties of the TIP4P water octamer
}

\author{
E. Asare, ${ }^{1}$ A-R. Musah, ${ }^{1}$ E. Curotto, ${ }^{1, a)}$ David L. Freeman, ${ }^{2}$ and J. D. Doll ${ }^{3}$ \\ ${ }_{1}^{1}$ Department of Chemistry and Physics, Arcadia University, Glenside, Pennsylvania, 19038-3295, USA \\ ${ }^{2}$ Department of Chemistry, University of Rhode Island, Kingston, Rhode Island 02881-1966, USA \\ ${ }^{3}$ Department of Chemistry, Brown University, Providence, Rhode Island 02912-9127, USA
}

(Received 29 January 2009; accepted 15 October 2009; published online 13 November 2009)

\begin{abstract}
Several stochastic simulations of the TIP4P [W. L. Jorgensen, J. Chandrasekhar, J. D. Madura, R. W. Impey, and M. L. Klein, J. Chem. Phys. 79, 926 (1983)] water octamer are performed. Use is made of the stereographic projection path integral and the Green's function stereographic projection diffusion Monte Carlo techniques, recently developed in one of our groups. The importance sampling for the diffusion Monte Carlo algorithm is obtained by optimizing a simple wave function using variational Monte Carlo enhanced with parallel tempering to overcome quasiergodicity problems. The quantum heat capacity of the TIP4P octamer contains a pronounced melting peak at $160 \mathrm{~K}$, about $50 \mathrm{~K}$ lower than the classical melting peak. The zero point energy of the TIP4P water octamer is $0.0348 \pm 0.0002$ hartree. By characterizing several large samples of configurations visited by both guided and unguided diffusion walks, we determine that both the TIP4P and the SPC [H. J. C. Berendsen, J. P. Postma, W. F. von Gunsteren, and J. Hermans, (Intermolecular Forces, Reidel, 1981). p. 331] octamer have a ground state wave functions predominantly contained within the $D_{2 d}$ basin of attraction. This result contrasts with the structure of the global minimum for the TIP4P potential, which is an $S_{4}$ cube. Comparisons of the thermodynamic and ground-state properties are made with the SPC octamer as well. () 2009 American Institute of Physics.
\end{abstract} [doi:10.1063/1.3259047]

\section{INTRODUCTION}

Neutral water clusters have been the focus of intense research for over three decades. A number of studies have focused on locating important low-lying minima and computing binding energies. ${ }^{1-22}$ Equal effort has been directed toward the simulations of thermodynamic and spectroscopic properties. ${ }^{23-43}$ Central to all these investigations is the issue of modeling, as accurately as possible, the potential energy surface. A number of potential energy surface models have been proposed. For example, the MCY (Matsuoka Clementi Yoshimine) potential ${ }^{25}$ is among the earliest models derived by fitting a number of configuration interaction computations on the dimer. A number of empirical or semiempirical potentials are obtained by fitting experimental and simulated properties of liquid water ${ }^{44-51}$ or ice. ${ }^{52,53}$

In the present work, we focus on the water octamer for two main reasons. First, a good deal of information is available in the literature about the water octamer. Second, its global minimum is a cubic structure, making it the smallest of the clusters with a compact and relatively highly symmetric global minimum. As such, the water octamer serves as an ideal testbed for theories and numerical methods for the simulation of more complex systems that contain water. ${ }^{54-62}$

Despite the relatively small size of the water octamer, its global optimization has proved to be challenging. Stillinger and David $^{3}$ report the first optimized structure of the octamer using a model that includes polarization corrections. Brink

${ }^{a)}$ Electronic mail: curotto@arcadia.edu. and Glasser ${ }^{4}$ report quite a different global minimum, cubic in shape with $D_{2 d}$ symmetry. Wawak et al. ${ }^{7}$ also find a global minimum with $D_{2 d}$ symmetry using the MCY potential. ${ }^{2}$ On the other hand, Tsai and Jordan, ${ }^{9}$ and independently, Wales and Ohmine, ${ }^{8}$ find two competing cubic structures, lying close in energy, one with $D_{2 d}$, and one with $S_{4}$ symmetry. Two of the potential surfaces tested by Wales and Ohmine ${ }^{8}$ are four-site models and favor the $S_{4}$ isomer energetically. The other two are three-site models and favor the $D_{2 d}$ cube instead. Hartke ${ }^{17}$ uses the TIP4P (Ref. 50) potential (a foursite model) and the genetic algorithm to optimize water clusters with up to 21 molecules. In the latter work, it is reported that clusters up to $n=10$ are solvable with one generation, demonstrating the power of the genetic algorithm. Nevertheless, the issue of which of the two low-lying cubes, the $D_{2 d}$ and the $S_{4}$, has the lowest energy is related to the quality of the potential energy model itself. High level ab initio computations of the binding energies of the two isomers have been performed. ${ }^{32}$ The difference between the binding energies of the two isomers is smaller than the error in the best potential energy model fitted to the ab initio points, and smaller than the estimated correlation effects beyond the second-order Møller Plesset (MP2) complete basis set limit. ${ }^{32}$ Therefore, to resolve the differences between the two minima, theories more accurate than the MP2 limit are necessary. The general consensus is that TIP4P approximates much better the true potential energy surface compared to the SPC or SCP/E, models. However, it is known that to get relative energies closer to the MP2 complete basis set limit 
results, one needs to include polarizable models.

Despite the daunting difficulties encountered in the formulation of a reliable potential energy model for the octamer, a number of simulations of the water octamer have been published. The characteristic vibrational frequencies have been computed with two different potentials. ${ }^{6,13}$ Thermodynamic properties have been computed in the NVT ensemble, ${ }^{23,28}$ in the NPT ensemble, ${ }^{29}$ and in the presence of external electric fields. ${ }^{41}$ Isomerizations and the melting transition have been studied using molecular dynamics. ${ }^{27}$ The bulk of the simulations of the water octamer in the literature have treated the motion of the nuclei classically. Yet, large quantum effects can be expected given the light mass of the nuclei involved. The intermolecular contributions of the zero point energy for the $D_{2 d}$ and the $S_{4}$ configurations have been computed with the rigid body diffusion Monte Carlo (DMC) ${ }^{13}$ whereas the equilibrium properties of the quantum water octamer have been simulated with the variational Gaussian wave packet method. ${ }^{43}$

One of our groups has been involved in the development of quantum methods for the efficient simulation of molecular aggregates. ${ }^{63-71}$ Constraining intramolecular degrees of freedom proves crucial in overcoming convergence difficulties with path integral Monte Carlo (PIMC) and DMC simulations of molecular clusters. The time scale difference between the intramolecular and the intermolecular degrees of freedom in typical molecular aggregates is sufficiently large to cause inefficiencies in DMC simulations, and to make the convergence of PIMC simulations a formidable task. ${ }^{67}$ In a recent study we use a relatively simple harmonic model for condensed matter with typical force constants for the intermolecular and intramolecular degrees of freedoms and analytical finite Trotter-number solutions of the path integral to quantify the efficiency gains of path integral simulations of molecular matter with holonomic constraints. ${ }^{67}$ For a difference in the values of the intramolecular and intermolecular spring constants as small as two orders of magnitude, constraining the high frequency degrees of freedom increases the numerical efficiency of the path integral substantially for both linear and cubically convergent estimators. ${ }^{67}$ Similar gains are found in another recent study aimed at developing DMC strategies for systems constrained holonomically. ${ }^{72}$

Holonomic constraints produce curved, non-Euclidean spaces. Consequently, the successful formulation of PIMC and DMC algorithms is highly dependent on the choice of coordinates. Using angular variables in PIMC simulations ${ }^{48,51,73-78}$ is possible only with the time-sliced formulations, and by neglecting precession effects for nonspherical tops. The latter approximation produces linearly convergent path integral methods. Precession effects contribute to second order. A number of approaches for rigid body DMC simulation methods can be found in the literature. ${ }^{79-86}$ Virtually all of these use schemes to avoid angular variables altogether. Clearly, variational (VMC) and DMC are much simpler in flat manifolds. ${ }^{87-102}$ The works we have cited are applications of VMC and DMC to clusters in Cartesian coordinates, and constitute only a small sample of the most recent investigations. On the other hand, diffusion in manifolds remains a topic of research in pure mathematics as well as mathematical physics. ${ }^{103}$

In both PIMC ${ }^{63-70}$ and $\mathrm{DMC}^{71,72}$ simulations in curved spaces, we find it highly advantageous to make use of stereographic projections. Our methods based on stereographic projection coordinates have been tested on a number of simple systems solvable by basis set methods, and several molecular clusters. ${ }^{64,67,69}$ We extend the reweighted random series method ${ }^{104-110}$ and the finite difference estimators ${ }^{108}$ for cubically convergent path integral simulations to manifolds. ${ }^{68}$ DMC simulations are nearly as straightforward to implement in curved spaces mapped with stereographic projection coordinates as they are with Cartesian coordinates. ${ }^{72}$ As the only difference, the distribution of the diffusion step in curved spaces is generally not a simple Gaussian, and several straight-forward rejection techniques have been tested successfully. ${ }^{71}$ Water clusters in the dimer through the octamer range have already served to gauge the feasibility of the stereographic projection path integral (SPPI) methods. ${ }^{68}$ Recently, we have explored the possibility to control the adiabatic approximation for water by allowing for couplings with high frequency modes. ${ }^{70}$

In a recent article we develop and test several importance sampling strategies for stereographic projection DMC in curved manifolds. ${ }^{72}$ In order to guide DMC, we propose a family of one parameter trial wave functions for VMC in stereographically projected manifolds. We find that guided DMC in manifolds is feasible, is orders of magnitude more efficient compared to unguided DMC, can overcome problems with nonconfining potentials, and can suppress quasiergodicity effectively. In particular, we find the Green's function stereographic projection DMC (GF-SPDMC) approach to have the simplest implementation and the best statistical behavior. $^{72}$

The purpose of the work reported in the present article is to determine the quantum thermodynamic and ground state properties of the TIP4P water octamer. Specifically, we formulate the following two sets of goals:

(i) obtaining the quantum and classical heat capacity of the TIP4P water octamer and comparing these quantities to the corresponding ones for the SPC water octamer from previous variational ${ }^{43}$ and path integral simulations. ${ }^{67}$ In Ref. 67 we use a three-site SPC model with a flexible extension ${ }^{49}$ identical to the one used by Frantsuzov. ${ }^{43}$

(ii) computing the ground state energy and characterizing structurally as much as possible the ground state wave function. The distributions of random walkers from guided DMC are for the product $\psi_{0} \psi_{T}$, where $\psi_{T}$ is the variational wave function used to guide DMC and $\psi_{0}$ is the true ground state wave function. By comparing distributions of $\psi_{0} \psi_{T}$, with those for $\left|\psi_{T}\right|^{2}$, we wish to obtain qualitative, but important information about the structural nature of the true ground state wave function $\psi_{0}$.

The rationale for the first set of goals is as follows. The TIP4P model is a four-site model for water, therefore one 
would anticipate significant differences in the thermodynamic properties when comparing with the SPC model for water. Given the difficulties encountered while trying to resolve which hydrogen bonding arrangement is favored energetically for the real water octamer by potential models, it is highly likely that future simulations of more complicated systems, such as clathrates for example, ${ }^{54-62}$ will require reproducibility tests of quantitative results with two or more models. The classical heat capacity of the octamer has proved to be sensitive to the details of the potential energy surface. $^{23,28,29,41}$ Little is known about how the quantum heat capacity and energy change when models of the potential energy vary.

The rationale for the second set of goals is similar. The sensitivity to small variations in the shape of the potential in classical simulations is probably exacerbated by the presence of the two low-lying cubes, which are nearly degenerate. In larger clusters, the number of nearly degenerate minima configurations with different arrangements of the hydrogen bond grows significantly. ${ }^{17}$ In ice, these different arrangements are nearly degenerate and give rise to a residual entropy as the temperature approaches $0 \mathrm{~K} .^{111,112}$ These complications make the simulations of physical properties of large water clusters and solid bulk water systems extremely challenging. It is our hope that the characterization of the ground state wave function of the water octamer can offer a glimpse into the role that quantum effects play in determining important simulated properties. In particular, we pose ourselves the following fundamental question: If certain hydrogen bond arrangements are nearly degenerate, is it possible that the smearing of the hydrogen atoms by quantum fluctuations in the ground state, and by thermal effects, is sufficiently extensive to wash out largely any differences predicted by slightly different numerical models of the potential energy surfaces? It is reasonable to expect that different properties in the ground state will be more sensitive to small changes than others, and that different size clusters will behave differently. Therefore, the scope of the investigation necessary to answer with some generality this question is greater than what we can accomplish with the present study. Nevertheless, our result should serve to lay the path for future investigations.

Lastly, both the SPPI and GF-SPDMC are relatively recent developments. It would be reassuring and instructive to find evidence that both approaches yield consistent results. Therefore, we compare the ground state energy obtained by the rigid body DMC against its value estimated with SPPI, and the finite difference estimator at several low temperatures.

The rest of the article is composed of three sections. All our simulation methods are in Sec. II. The results are presented and discussed in Sec. III, and our conclusions are in Sec. IV.

\section{SIMULATION METHODS}

\section{A. Stereographic projection path integral}

In a series of works ${ }^{63-70}$ we have extended the reweighted random series approach ${ }^{104-110}$ to the imaginary time path integral in manifolds that are mappable by stereo- graphic projections. The random series expansion of the path consists of $k_{m}$ core terms and an additional $3 k_{m}$ tail terms,

$$
\widetilde{q}^{\mu}(u)=q_{r}^{\mu}(0)+\hbar \beta^{1 / 2} \sum_{k=1}^{k_{m}} a_{k}^{\mu} \Lambda_{k}(u)+\hbar \beta^{1 / 2} \sum_{k=k_{m}+1}^{4 k_{m}} a_{k}^{\mu} \tilde{\Lambda}_{k}(u),
$$

$\widetilde{q}^{\mu}$ is the closed path $\left(\widetilde{q}^{\mu}(0)=\widetilde{q}^{\mu}(1)\right)$ with $u=\tau / \beta \hbar$. The tail terms are formulated in such a way that the partial averaging method constructed on Eq. (1) is identical to the partial averaging method derived from the original random series representation of the Brownian bridge. ${ }^{104-110}$

The centerpiece of the imaginary time path integral approach is the density matrix $\rho\left(q, q^{\prime}, \beta\right)$. In manifolds the following equation is convenient for developing the proper importance sampling algorithms, ${ }^{113-115}$ and to derive the expressions for the finite difference estimators of the energy and heat capacity,

$$
\begin{aligned}
\rho\left(q, q^{\prime}, \beta\right)= & \left(\frac{1}{2 \pi}\right)^{N D / 2}\left(\hbar^{2} \beta\right)^{-D / 2} J_{\Lambda} \\
& \times \int d[a]_{r} \exp \left\{-\beta \int_{0}^{1} d u U\left(\widetilde{q}^{\mu}(u)\right)\right\},
\end{aligned}
$$

where $U\left(\widetilde{q}^{\mu}(u)\right)$ is the quantum action,

$$
\begin{aligned}
U\left(\widetilde{q}^{\mu}(u)\right)= & -\frac{N}{2 \beta} \ln \left[\operatorname{det} g_{\mu \nu}\left(\widetilde{q}^{\mu}(u)\right)\right] \\
& +\frac{1}{2} g_{\mu \nu}\left(\widetilde{q}^{\mu}(u)\right) \dot{q}^{\mu} \dot{q}^{\nu}+V\left(\widetilde{q}^{\mu}(u)\right),
\end{aligned}
$$

$d[a]_{r}$ is the measure of the space of the series coefficients,

$$
d[a]_{r}=\prod_{k=1}^{4 k_{m}} \prod_{\mu=1}^{D} d a_{k}^{\mu}
$$

and $J_{\Lambda}$ is a constant Jacobian ${ }^{67}$ that results from transforming the Wiener measure over the time-sliced space to the space of all the random coefficients $a_{k}^{\mu}$.

In Eq. (3), $g_{\mu \nu}$ is the Hessian metric tensor, ${ }^{116,117}$ which can be represented with a block-diagonal matrix, ${ }^{67}$

$$
g_{\mu \nu}=\left(\begin{array}{ccc}
\mathbf{g}^{(1)} & 0 & 0 \\
\ldots & \ldots & \ldots \\
0 & 0 & \mathbf{g}^{(n)}
\end{array}\right) .
$$

Each block in Eq. (5) represents the molecular metric tensor, a $6 \times 6$ block-diagonal matrix,

$$
\mathbf{g}^{(i)}=\left(\begin{array}{cccc}
m & 0 & 0 & \\
0 & m & 0 & \mathbf{0} \\
0 & 0 & m & \\
& \mathbf{0} & & \mathbf{G}^{(i)}
\end{array}\right),
$$

where $m$ is the total mass of the water molecule and $\mathbf{G}^{(i)}$ is the metric tensor associated with the orientations.

To compute the integral in Eq. (2), we use the trapezoid rule with $N=k_{m}+1$ points, and we evaluate the Hessian metric tensor and the potential at the end point of every interval $u_{j}=j / N$. The end point choice yields a constant curvature for 
the quantum Jacobian that we can simply ignore. ${ }^{18}$ Additionally, we can ignore the difference between the Jacobian obtained from the manifold and the Jacobian obtained in $\mathbb{R}^{9 n}$, with infinitely stiff springs replacing the holonomic constraints, ${ }^{115}$ since their ratio is a constant. ${ }^{70}$ To implement the Metropolis algorithm, we move the center of mass or the orientation of a randomly selected molecule, and we translate one path variable for each coordinate moved, e.g., $a_{k}^{6(i-1)+1}, a_{k}^{6(i-1)+2}, a_{k}^{6(i-1)+3} \rightarrow\left(a_{k}^{6(i-1)+1}, a_{k}^{6(i-1)+2}, a_{k}^{6(i-1)+3}\right)^{\prime}$. The integer $k$ is in $\left(1 \leq k \leq 4 k_{m}\right)$, and is selected randomly from a uniform distribution.

The estimators for the energy and heat capacity are computed using the following two expressions, respectively, ${ }^{67}$

$$
\begin{aligned}
\langle E\rangle_{\beta}= & \frac{D}{2 \beta}+\left\langle\frac{\partial}{\partial \beta}\left[\beta \int_{0}^{1} d u U\left(\widetilde{q}^{\mu}(u)\right)\right]\right\rangle, \\
\frac{C_{V}}{k_{B}}= & \frac{D}{2}+\frac{D^{2}}{4}+D \beta\left\langle\frac{\partial}{\partial \beta}\left[\beta \int_{0}^{1} d \tau U\left(\widetilde{q}^{\mu}(u)\right)\right]\right\rangle \\
& +\beta^{2}\left\langle\left\{\frac{\partial}{\partial \beta}\left[\beta \int_{0}^{1} d u U\left(\widetilde{q}^{\mu}(u)\right)\right]\right\}^{2}\right\rangle \\
& -\beta^{2}\left\langle\frac{\partial^{2}}{\partial \beta^{2}}\left[\beta \int_{0}^{1} d u U\left(\widetilde{q}^{\mu}(u)\right)\right]\right\rangle \\
& -\left\{-\frac{D}{2}-\beta\left\langle\frac{\partial}{\partial \beta}\left[\beta \int_{0}^{1} d u U\left(\widetilde{q}^{\mu}(u)\right)\right]\right\rangle\right\}^{2} .
\end{aligned}
$$

The derivatives with respect to $\beta$ in Eqs. (7) and (8) are evaluated numerically. Previous work on SPC water clusters $^{67}$ has indicated that SPPI simulations with the Fourier-Wiener reweighted random series are converged with $k_{\max }=32$ at and above $50 \mathrm{~K}$ for the energy, and above $100 \mathrm{~K}$ for the heat capacity.

The potential energy used in our simulations is the TIP4P (Ref. 50) model, added to a smooth Lee-BarkerAbraham spherical cavity, ${ }^{118}$

$$
V_{\mathrm{LBA}}=V_{0} \sum_{i=1}^{8}\left(\frac{\mathbf{r}_{\mathrm{O}, i}-\mathbf{R}_{C M}}{2 R_{C}}\right)^{20},
$$

where $\mathbf{r}_{\mathrm{O}, i}$ is the location of the oxygen atom for the $i$ th water molecule, $\mathbf{R}_{C M}$ is the center of mass of the cluster, $V_{0}=1$ hartree, and $R_{C}$ is $12.0 \mathrm{bohr}$.

\section{B. Green's function stereographic projection diffusion Monte Carlo}

The integral representation of the imaginary timedependent Schrödinger equation is the starting point of the GF-SPDMC,

$$
\psi(q, \tau+\Delta \tau)=\int_{\mathbb{M}} G\left(q, q^{\prime}, \Delta \tau\right) \psi\left(q^{\prime}, \tau\right) d q^{\prime} .
$$

In Eq. (10), $M$ is the manifold and $G\left(q, q^{\prime}, \tau\right)$ is the Green's function. $G\left(q, q^{\prime}, \tau\right)$ satisfies the imaginary time dependent Schrödinger equation,

$$
\frac{\partial G}{\partial \tau}=\frac{\hbar^{2}}{2} \Delta_{\mathrm{LB}} G-\left(V-E_{T}\right) G,
$$

and the initial condition

$$
\lim _{\Delta \tau \rightarrow 0} G\left(q, q^{\prime}, \Delta \tau\right)=\delta\left(q-q^{\prime}\right) .
$$

In Eq. (11), $\Delta_{\mathrm{LB}}$ is the Laplace-Beltrami operator, ${ }^{117}$

$$
\Delta_{\mathrm{LB}}=\frac{1}{\sqrt{g}} \partial_{\nu} g^{\mu \nu} \sqrt{g} \partial_{\mu},
$$

where $g^{\mu \nu}$ is the inverse of the Hessian metric tensor, and $g$ is the determinant of $g_{\mu \nu}$. Equation (10) can be solved recursively ${ }^{87}$ by approximating the Green function locally and introducing an optimized trial wave function $\psi_{T}$,

$$
\exp \left(-\frac{g_{\mu \nu} \Delta q^{\mu} \Delta q^{\nu}}{2 \Delta \tau}\right) \frac{\psi_{T}(q+\Delta q)}{\psi_{T}(q)} \exp \left[-\left(V-V_{\text {ref }}\right) \Delta \tau\right],
$$

where $\psi_{T}(q)$ and $\psi_{T}(q+\Delta q)$ are the values of the optimized trial wave function before the diffusion step is taken and after the diffusion step is taken, respectively. Each replica is moved with $\Delta q$ distributed according to

$$
\exp \left(-\frac{g_{\mu \nu} \Delta q^{\mu} \Delta q^{\nu}}{2 \Delta \tau}\right)
$$

and grown or annihilated using

$$
w_{i}=\frac{\psi_{T}(q+\Delta q)}{\psi_{T}(q)} \exp \left[-\left(V-V_{\mathrm{ref}}\right) \Delta \tau\right],
$$

as the statistical weight. The spacial dependence of the Hessian metric tensor in Eq. (15) complicates slightly the diffusion part of the algorithm. Several sensible methods have been tested in this regard, ${ }^{71}$ using a number of different test problems. All the schemes are found to converge to first $\operatorname{order}^{72}$ in $\Delta \tau$.

The GF-SPDMC strategy needs a trial wave function in M. In Ref. 72, we explore the following possibility:

$$
\psi_{T}(q)=A \exp \left\{-\frac{1}{2} \alpha V(q)\right\},
$$

using the bistable potential and the Stockmayer trimer. The variational energy,

$$
\left\langle E_{\alpha}\right\rangle=\frac{\int_{\mathbb{M}} d q g^{1 / 2}\left(\psi_{T}^{-1} \hat{H} \psi_{T}\right)\left|\psi_{T}\right|^{2}}{\int_{\mathbb{M}} d q g^{1 / 2}\left|\psi_{T}\right|^{2}},
$$

is computed with parallel tempering ${ }^{119-122}$ for various values of $\alpha$. With the form of $\psi_{T}$ given in Eq. (17), $\left|\psi_{T}\right|^{2}$ is the equivalent of the importance sampling function for the classical configuration integral of statistical mechanics,

$$
\left|\psi_{T}\right|^{2}=A^{2} \exp \{-\alpha V(q)\},
$$

where $\alpha$ plays the same role as $\left(k_{B} T\right)^{-1}$. The wave function in Eq. (17) has several desirable features. First, it is the exact wave function for a harmonic monodimensional system. Second, it produces a convenient importance sampling function for the most important regions of configuration space. When the parameter $\alpha$ is optimized, we find that $\psi_{T}$ usually samples a multitude of potential energy minima decreasing 
the possibility that the guided diffusion walk is biased toward one single minimum of the potential energy surface. Since the random walk performed to optimize $\psi_{T}$ is carried out using parallel tempering, starting the diffusion walk from a distribution obtained at the optimized parameter with parallel tempering gives us confidence that quasiergodicity is reduced in the diffusion walk. In the unguided DMC case, quasiergodicity manifests itself with relatively long correlation times when all the replicas become trapped in one well. $^{72}$ Third, it reduces the importance of dissociated or nearly dissociated states when the potential surface is not confining, as is usually the case with clusters. Finally, the procedure conveniently produces an optimized wave function while computing thermodynamic properties of interest in the classical limit, and ensuring ergodic sampling in $\mathbb{M}$ at the same time.

Using Eqs. (13) and (17), one derives the following expression for the local energy estimator:

$$
\begin{aligned}
\Psi_{T}^{-1} \hat{\mathcal{H}} \Psi_{T}= & V-\frac{\hbar^{2}}{2}\left\{\frac{\alpha^{2}}{4} g^{\mu \nu} \partial_{\mu} V \partial_{\nu} V-\frac{\alpha}{2} g^{\mu \nu} \partial_{\mu} \partial_{\nu} V\right. \\
& \left.-\frac{\alpha}{2}\left\{g^{\mu \nu}\left[\partial_{\mu} \ln \sqrt{\operatorname{det}(g)}\right]+\left(\partial_{\mu} g^{\mu \nu}\right)\right\} \partial_{\nu} V\right\} .
\end{aligned}
$$

Equation (20) is used to estimate the energy from populations evolved according to the GF-SPDMC algorithm as well. The trial wave function $\psi_{T}$ given in Eq. (17) represents the compromise we make to embody all the physics of the system, at the expense of the computer time necessary to evaluate the derivatives in Eq. (20).

\section{Structural characterization methods}

The comparison between two configurations of a cluster is carried out with the structural comparison algorithm ${ }^{123}$ (SCA), which has been modified for the present work. The algorithm to compare configuration $A$ and $B$ is as follows. Configurations $A$ and $B$ are translated so that the origin is the cluster's geometric center,

$$
\sum_{i=1}^{3 n} \mathbf{r}_{i}^{(A)}=\mathbf{0}, \quad \sum_{i=1}^{3 n} \mathbf{r}_{i}^{(B)}=\mathbf{0} .
$$

Configurations $A$ and $B$ are rotated so that atom 1 and $i$, respectively, are on the $z$ axis $(1 \leq i \leq 3 n)$. One additional rotation is performed so that atom 2 and $j$, for configurations $A$ and $B$, respectively, are on the $x-z$ plane $(1 \leq j \leq 3 n, j$ $\neq i)$. For each of the $3 n(3 n-1)$ rotations, a sorting of the remaining $3 n-2$ atoms is performed to find the atom of $B$ that is the closest to atom $k$ of $A$, for $k=2, \ldots, 3 n$. The SCA metric is the infimum of the set,

$$
\Delta_{A}=\inf \left\{\Delta_{i j}^{(A)}\right\}_{i, j=1, i \neq j}^{3 n},
$$

where $\Delta_{i j}^{(A)}$ represent an element of the following set of sums,

$$
\Delta_{i j}^{(A)}=\sum_{k=1}^{3 n}\left[\mathbf{r}_{k}^{(A)}-\mathcal{P} \mathbf{R}\left(\Omega_{i}, \Omega_{j}\right) \mathbf{r}_{k}^{(B)}\right],
$$

and the element of the set $\left\{\mathcal{P} \mathbf{R}\left(\Omega_{i}, \Omega_{j}\right) \mathbf{r}_{k}^{(B)}\right\}_{k=1}^{3 n}$ is the configuration $B$ rotated and with the labels permuted as described.
The quantity $\Delta_{A}$ is most meaningful when the configuration $A$ is a minimum of the potential energy surface. If $A$ is the $S_{4}$ cube we use the symbol $\Delta_{S 4}$, and if $A$ is the $D_{2 d}$ cube we use the symbol $\Delta_{D 2 d}$. The atoms are all treated as identical, namely, no distinction is made between the hydrogen and the oxygen atoms. We refer to the metric in Eq. (22) as the all-atoms SCA distance.

Previous applications of the SCA to molecular clusters involved only the Cartesian coordinates of the centers, unlike those of every atom as we do here. The measure $\Delta_{A}$ in the present version is sensitive to changes in the orientations of the water molecules. However, for the purpose of the structural analysis in the present work we find it necessary to introduce an additional SCA measure based on the oxygen atoms alone,

$$
\Delta_{A}^{(\mathrm{O}-\mathrm{O})}=\inf \left\{\Delta_{(A), i j}^{(\mathrm{O}-\mathrm{O})}\right\}_{i, j=1, i \neq j}^{n},
$$

where $\Delta_{(A), i j}^{(\mathrm{O}-\mathrm{O})}$ is defined using an equation analogous to Eq. (23),

$$
\Delta_{(A), i j}^{(\mathrm{O}-\mathrm{O})}=\sum_{k=1}^{n}\left[\mathbf{r}_{\mathrm{O}, k}^{(A)}-\mathcal{P} \mathbf{R}\left(\Omega_{i}, \Omega_{j}\right) \mathbf{r}_{\mathrm{O}, k}^{(B)}\right],
$$

and $\mathbf{r}_{\mathrm{O}, k}^{(A)}$ is the position of the $k$ th oxygen atom relative to the geometric center of the cluster. Using this strategy, we can determine how translational degrees of freedom are distributed differently compared with the orientation degrees of freedom in the GF-SPDMC and the VMC ground state. Differences in these distributions from quantum effects are expected given that the effective mass associated with translations is approximately a factor of 10 larger than the effective masses associated with the orientation of the molecules. When $A$ is the $S_{4}$ minimum we abbreviate $\Delta_{A}^{(\mathrm{O}-\mathrm{O})}$ (what we call the O-atoms SCA distance) with the symbol $\Delta_{S 4}^{(\mathrm{O}-\mathrm{O})}$.

\section{Quenching in curved manifolds}

To confirm further the results obtained from the structural analysis, we submit configurations collected during the random walks to a quenching procedure. The method we choose is a $0 \mathrm{~K}$ Brownian algorithm, which we have recently developed to study ammonia clusters. To handle the holonomic constraints we begin with the Euler-Lagrange's equations,

$$
\frac{d}{d t}\left(\frac{\partial \mathcal{L}}{\partial \dot{q}^{\nu}}\right)-\left(\frac{\partial \mathcal{L}}{\partial q^{\nu}}\right)=0 .
$$

These are invariant under any continuous change of coordinates, and therefore are applicable in any manifold produced by holonomic constraints. From Eq. (26) we could derive the geodesic equations for accelerated systems in manifolds ${ }^{116}$ by rewriting the derivative of the Hessian metric tensor into a symmetrized version, known as the Christoffel connection coefficients. However, the connection coefficients ${ }^{116}$ in ellipsoid of inertia mapped stereographically have formidable expressions. Instead, we start with the general following form of the Lagrangian, 


$$
\mathcal{L}=\frac{1}{2} g_{\mu \nu} \dot{q}^{\mu} \dot{q}^{\nu}-V
$$

where $V$ is the potential energy and $g_{\mu \nu}$ is the Hessian metric tensor, and insert this into Eq. (26). This yields the following expression:

$$
g_{\mu \nu} \ddot{q}^{\mu}+\left(\partial_{\nu} g_{\mu \beta}\right) \dot{q}^{\mu} \dot{q}^{\beta}-\frac{1}{2}\left(\partial_{\nu} g_{\mu \beta}\right) \dot{q}^{\mu} \dot{q}^{\beta}+\partial_{\nu} V=0 .
$$

The first two terms in Eq. (28) result from the time derivative of the partial of $\mathcal{L}$ with respect to $\dot{q}^{\nu}$. A trivial rearrangement yields the equivalent of Newton's second law in manifolds,

$$
g_{\mu \nu} \ddot{q}^{\mu}=-\partial_{\nu} \mathcal{L}^{+},
$$

where $\mathcal{L}^{+}$is the Lagrangian with the potential inverted,

$$
\mathcal{L}^{+}=\frac{1}{2} g_{\mu \nu} \dot{q}^{\mu} \dot{q}^{\nu}+V .
$$

Equation (29) is the starting point for our algorithm. The acceleration vector is

$$
\ddot{q}^{\mu}=-g^{\mu \nu} \partial_{\nu} \mathcal{L}^{+} .
$$

We integrate Eq. (31) assuming that $\left(g^{\mu \nu} \partial_{\nu} \mathcal{L}^{+}\right)_{n}$ is constant for sufficiently small values of $\Delta t$. Given the initial conditions $\left(q^{\mu}, \dot{q}^{\mu}\right)_{n}$ for step $n=0,1, \ldots$, we compute $\partial_{\nu} \mathcal{L}_{n}^{+}$by finite difference for $\nu=1,2, \ldots, d$. We then compute the inverse of the Hessian metric tensor $g^{\mu \nu}$ and the product $\left(g^{\mu \nu} \partial_{\nu} \mathcal{L}^{+}\right)_{n}$. Assuming that $\left(g^{\mu \nu} \partial_{\nu} \mathcal{L}^{+}\right)_{n}$ is constant, we can integrate and update the velocities,

$$
\left(\dot{q}^{\mu}\right)_{n+1}=\left(\dot{q}^{\mu}\right)_{n}-\left(g^{\mu \nu} \partial_{\nu} \mathcal{L}^{+}\right)_{n} \Delta t-\gamma\left(\dot{q}^{\mu}\right)_{n} \Delta t,
$$

where $\gamma$ is the usual drag coefficient. Using a similar assumption for the velocities, we update the configuration using

$$
\left(q^{\mu}\right)_{n+1}=\left(q^{\mu}\right)_{n}+\left(\dot{q}^{\mu}\right)_{n+1} \Delta t .
$$

At every step the energy is computed with $E\left(t_{n+1}\right)=\left(\mathcal{L}^{+}\right)_{n+1}$. Although we use stereographic projections in this work, the algorithm can be implemented as written with all types of regular coordinate transformations (those derived from analytical maps), such as Euler angles, quaternions, and so on. With stereographic projections, occasionally large values are produced, and the code needs to trap and recover from the event otherwise floating exceptions occur. We do this simply by changing the sign of the projection and rescaling its velocity whenever the absolute value of a projection is larger than an arbitrarily set upper limit. Additionally, it is straight forward to derive a Verlet-like algorithm from Eq. (29), which is useful whenever constant energy dynamics are required.

\section{RESULTS}

\section{A. Thermodynamic and ground state properties}

The outcome of the SPPI calculations, both for $k_{\max }=0$ [classical, cf. Eq. (1)] and $k_{\max }=32$ (quantum) are presented in Figs. 1(a) and 1(d). Figure 1(a) is a graph of the energy in the classical (white squares) and quantum limit (black squares) in hartree, as functions of the temperature. The error bars in Figs. 1(a), 1(b), and 1(d) are obtained by computing a 95\% confidence interval from ten independent parallel tempering ${ }^{119-122}$ simulations. Each simulation consists of $10^{7}$
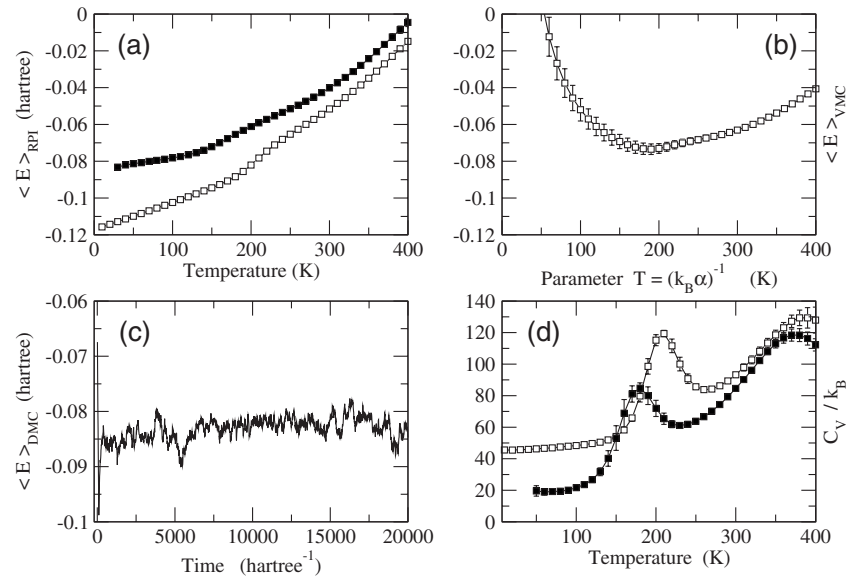

FIG. 1. (a) Energy of the TIP4P water octamer as a function of temperature. The white squares are the classical simulation data, the gray squares are the quantum results $\left[k_{m}=0\right.$ and $k_{m}=32$, respectively, in Eq. (7)]. (b) Variational ground state energy of the water TIP4P octamer in hartree graphed as a function of the parameter $T=\left(k_{B} \alpha\right)^{-1}$ [cf. Eq. (20)]. (c) Ground state energy estimate from a single GF-SPDMC with a target population size of $10^{5}$ configurations and $\Delta \tau=10$ hartree $^{-1}$ [cf. Eq. (20)]. (d) Heat capacity of the TIP4P water octamer in units of the Boltzmann constant, as a function of temperature in kelvin. The white squares are the classical simulation data and the black squares are the quantum results $\left[k_{m}=0\right.$ and $k_{m}=32$ respectively, in Eq. (8)].

warm up moves, followed by an additional $10^{7}$ moves to accumulate physical property estimates. For the quantum heat capacity, we repeat a five block simulation twice, the second time starting from the previously equilibrated configurations. The results from the two simulations are statistically indistinguishable. A total of 40 walkers is used to implement the parallel tempering runs between the temperatures of 10 and $400 \mathrm{~K}$. The temperature values are equally spaced $10 \mathrm{~K}$ apart. In Ref. 67 we study the convergence of the SPPI using the SPC water octamer. We find that the $k_{\max }=32$ finite difference estimate of the energy converges within the statistical error at $50 \mathrm{~K}$ and above. The same applies for the finite difference estimator of the heat capacity at $100 \mathrm{~K}$ and above. Therefore, we do not deem it necessary to remap the convergence profile for the TIP4P water octamer in the present work. The classical energy [white squares in Fig. 1(a)] approaches the value of the global minimum as the temperature approaches $0 \mathrm{~K}$. The quantum energy is not converged below $40 \mathrm{~K}$, and the data points below $40 \mathrm{~K}$ have been excluded from the graph. At $40 \mathrm{~K}$, the value of the energy is $-0.0820 \pm 0.0002$ hartree. Inflections indicative of phase changes are visible in both curves. The centers of these features are at $210 \mathrm{~K}$ in the classical curve and $170 \mathrm{~K}$ in the quantum curve. The quantum effects are substantial even at $400 \mathrm{~K}$, although the pattern indicates that the two curves merge at higher temperatures. The differences between the classical and the quantum energy are in reasonable agreement with our recent computations of the SPC octamer. ${ }^{67}$

The heat capacities in units of the Boltzmann constant as a function of temperature in kelvin, obtained from the simulations are graphed in Fig. 1(d). The classical heat capacity approaches the equipartition value of 45 Boltzmann's constant units at low temperatures. For $n$ nonlinear rigid bodies 
the equipartition limit for the constant volume heat capacity is $(6 n-3) k_{B}$. Two features are visible in both curves. The peak at $380 \mathrm{~K}$ in the classical heat capacity is matched with one equally broad and nearly as intense at $370 \mathrm{~K}$ in the quantum heat capacity. The sharper melting peak at $210 \mathrm{~K}$ in the classical heat capacity is also matched with a broader and less intense feature centered around $160 \mathrm{~K}$. These two temperatures correlate closely with the inflections in the energy visible in Fig. 1(a). The heat capacity of the TIP4P octamer is quite different from that of the SCP octamer. ${ }^{67}$ The melting peak in the classical heat capacity at $140 \mathrm{~K}$ is "washed away" when the SCP potential is used. The quantum heat capacity for the TIP4P octamer in Fig. 1(d) has a well defined and relatively intense melting feature compared to the SCP model.

In Fig. 1(b) the variational energy in hartree computed using the methods in Sec. II B is graphed. The graph of $\left\langle E_{\alpha}\right\rangle$ is quadratic about the minimum at $190 \mathrm{~K}$, for a relatively narrow range of values of $T$, and it deviates significantly from a quadratic function at higher temperatures. The lack of symmetry in the $\left\langle E_{\alpha}\right\rangle$ curve is the consequence of the onset of a phase change that peaks around $210 \mathrm{~K}$. The minimum value of $\left\langle E_{\alpha}\right\rangle$ is $-0.074 \pm 0.002$ hartree at $190 \mathrm{~K}$. Figure $1(\mathrm{c})$ contains the graph of the population energy from a GFSPDMC calculation, with $N=10^{5}$ as the target size and with $\Delta \tau=10$ hartree $^{-1}$. The simulation is repeated ten times, and the energy values between 10000 and 20000 hartree $^{-1}$ are averaged for each independent run. The resulting block averages are used to estimate the ground state energy at $-0.0823 \pm 0.0002$ hartree. The statistical error is computed using the standard error in the mean. If one assumes that the system is predominantly in the ground state at and below $40 \mathrm{~K}$, then the energy for the $k_{\max }=32$ data in Fig. 1(a) should be a reasonable approximation of the ground state energy. The value of the energy $\langle E\rangle_{k_{m}=32}$ at $40 \mathrm{~K}$ is statistically indistinguishable from the DMC result, and the "exact" value of the ground state energy is $11 \%$ lower than the variational energy in Fig. 1(b).

\section{B. Structural analysis of the TIP4P octamer surface}

In order to build meaningful structural identifiers, we begin by requenching 1291 minima of the octamer, obtained in our previous work on water clusters. ${ }^{67}$ These structures were found using the SPC/F2 potential, ${ }^{49}$ and we expect to see some differences with the TIP4P potential. We find 933 distinct structures after requenching. The $S_{4}$ isomer is slightly lower in energy compared to the $D_{2 d}$ cube, which is consistent with previous comparisons between three-site and four-site potential energy models. ${ }^{8}$ All 933 minima configurations are measured using the all-atoms SCA against the $S_{4}$ cube, [cf. Eq. (22)] and the O-atoms SCA against the $S_{4}$ cube, [cf. Eq. (24)]. As explained in Sec. III A, we label these two measures $\Delta_{S 4}$ and $\Delta_{S 4}^{(\mathrm{O}-\mathrm{O})}$, respectively. Figure 2 contains two graphs of the measures $\Delta_{S 4}$ (left panel), and $\Delta_{S 4}^{(\mathrm{O}-\mathrm{O})}$ (right panel) plotted against the energy for all 933 distinct minima. Figure 2 gives us a qualitative glimpse at the complexity of the TIP4P surface for the octamer. The $\Delta_{S 4}$ measure spans a domain $60 \%$ larger than $\Delta_{S 4}^{(\mathrm{O}-\mathrm{O})}$. The $S_{4}$ and
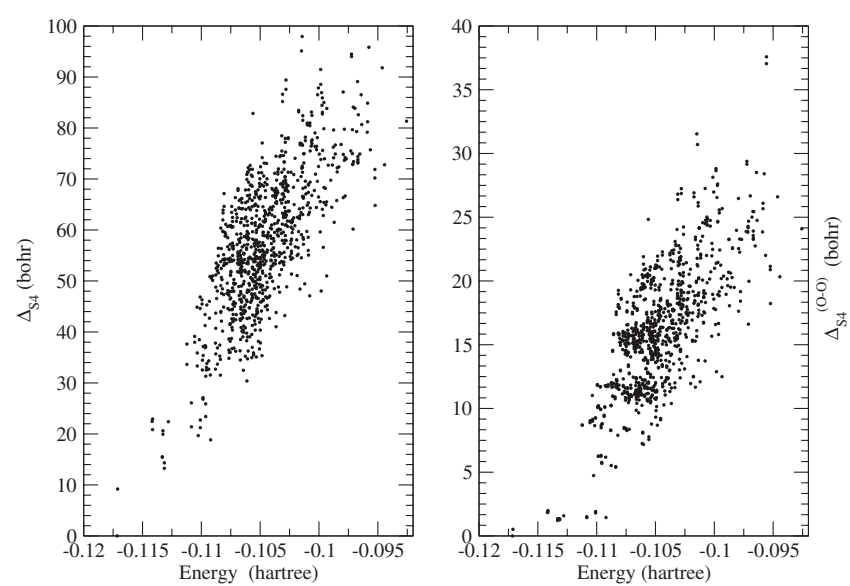

FIG. 2. Left graph: A plot of the all-atoms SCA distance [cf. Eq. (22)] from the $S_{4}$ isomer as a function of energy measured for 933 distinct minima of the TIP4P water octamer. Right graph: A plot of the O-atoms SCA distance from the $S_{4}$ isomer as a function of energy measured for 933 distinct minima of the TIP4P water octamer.

$D_{2 d}$ cubes are very close in energy and are followed by a group of nine minima that are cubic in shape. The lowest 11 minima, within $-0.1171<E<-0.1128$ hartree, can be easily identified in both the left and the right graph of Fig. 2. These 11 structures have $\Delta_{S 4}<24$ bohr and $\Delta_{S 4}^{(\mathrm{O}-\mathrm{O})}$ $<2$ bohr. An additional six cubes are located within $-0.1108<E<-0.1092$ hartree, $\Delta_{S 4}<24$ bohr, and $\Delta_{S 4}^{(\mathrm{O}-\mathrm{O})}$ $<2$ bohr. The majority of the minima have energies greater than -0.11 hartree, $\Delta_{S 4}>25$ bohr, and $\Delta_{S 4}^{(\mathrm{O}-\mathrm{O})}>2$ bohr. However, none of these are important for the thermodynamic and ground state properties.

\section{Structural analysis of the VMC walk}

With SCA we analyze several random walks at $\left(k_{B} \alpha\right)^{-1}$ $=190 \mathrm{~K}$. After the initial $10^{6}$ moves to reach the asymptotic limit, we store $10^{5}$ configurations to the disk from the walker at $190 \mathrm{~K}$. The computation is repeated ten times independently on ten separate nodes of our computer array. A total of $10^{6}$ configurations are measured in this manner, using SCA, against the $S_{4}$ cube and the $D_{2 d}$ cube. We create histograms of the $\Delta_{S 4}, \Delta_{D 2 d}$, and $\Delta_{S 4}^{(\mathrm{O}-\mathrm{O})}$ properties, using 1000 classes for the range. The distributions of $\Delta_{S 4}, \Delta_{D 2 d}$, and $\Delta_{S 4}^{(\mathrm{O}-\mathrm{O})}$ from the $190 \mathrm{~K}, k_{m}=0$, random walks can be found in Figs. 3-5 represented with black dots. The distributions obtained from the walks at $\left(k_{B} \alpha\right)^{-1}=190 \mathrm{~K}$ are projections of $\left|\Psi_{T}\right|^{2}$ along the $\Delta_{S 4}, \Delta_{D 2 d}$, and $\Delta_{S 4}^{(\mathrm{O}-\mathrm{O})}$ "coordinates."

The $\Delta_{S 4}$ and $\Delta_{D 2 d}$ distributions from the variational simulation at $\left(k_{B} \alpha\right)^{-1}=190 \mathrm{~K}$ have multiple features. For $\Delta_{S 4}$, in Fig. 3 , the variational simulation yields a distribution with a relatively intense peak around 20 bohr, a small shoulder at 12-14 bohr, and a broad and less intense feature between 38 and 76 bohr. For $\Delta_{D 2 d}$, in Fig. 4, the variational simulation yields a similar distribution; however, the most intense peak is located at 12 bohr, the small shoulder is around 20 bohr, and the broad and less intense feature between 38 and 76 bohr is nearly identical to its $\Delta_{S 4}$ counterpart in Fig. 3.

The distribution of the variable $\Delta_{S 4}^{(\mathrm{O}-\mathrm{O})}$ from the varia- 


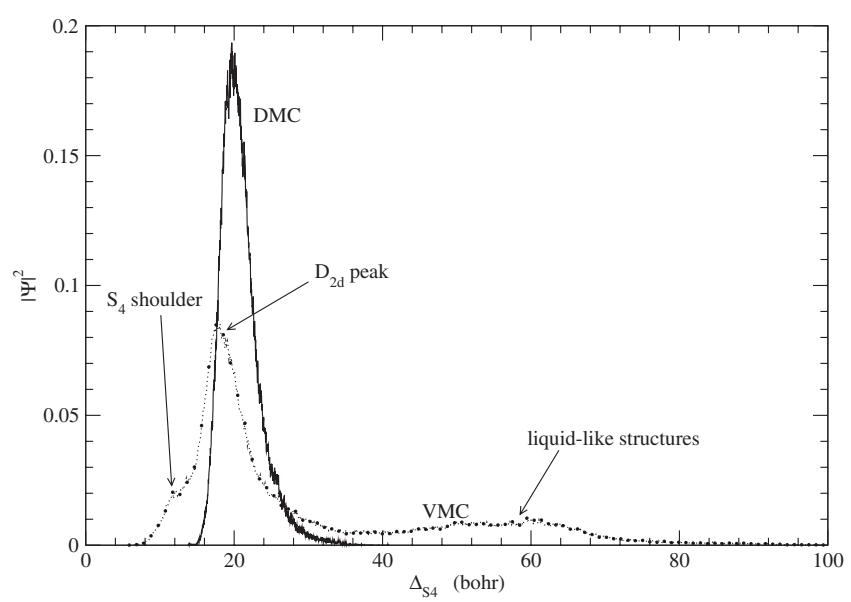

FIG. 3. A graph of the ground state density projected along the all-atoms SCA distance [cf. Eq. (22)] from the $S_{4}$ cube obtained from the VMC simulations (dots) and the GF-SPDMC simulation (lines).

tional simulation in Fig. 5 has only two features, an intense peak at $3 \mathrm{bohr}$, and a broad peak between 10 and $24 \mathrm{bohr}$. The distributions in Figs. 3-5 allow us to obtain a qualitative picture of the optimized variational wave function we have chosen to use and its accuracy. The most important feature of these distributions is the shoulder easily assigned to the presence of the $S_{4}$ cube. To be certain, we quench a sample of 245602 configuration from the variational walk. Upon quenching the VMC configuration sample, we obtain $58 \%$ of the replicas collecting in the $D_{2 d}$ minimum, approximately $30 \%$ in the $S_{4}$ basin, and the remaining $12 \%$ in higher energy isomers.

The broad peak at large values of $\Delta_{S 4}, \Delta_{D 2 d}$, and $\Delta_{S 4}^{(\mathrm{O}-\mathrm{O})}$ can be seen in all three graphs. In particular, the presence of the peak around 15 bohr in the $\Delta_{S 4}^{(\mathrm{O}-\mathrm{O})}$ distribution indicates that our $\left|\psi_{T}\right|^{2}$ distribution oversamples open configurations, or configurations other than the cube. The presence of molten states with respect to the molecular centers framework is responsible for the peaks around $60 \mathrm{bohr}$ in the distributions of $\Delta_{S 4}$ and $\Delta_{D 2 d}$ from the variational walk. The features in the distributions of the three SCA metrics disappear in the DMC walk. The oversampling of the variational walk of

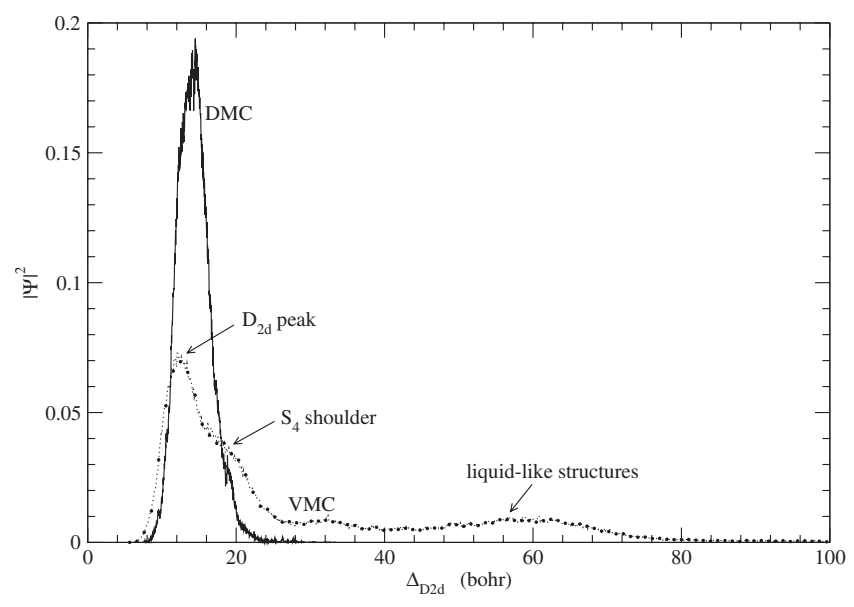

FIG. 4. A graph of the ground state density projected along the all-atoms SCA distance [cf. Eq. (22)] from the $D_{2 d}$ cube obtained from the VMC simulations (dots) and the GF-SPDMC simulation (lines).

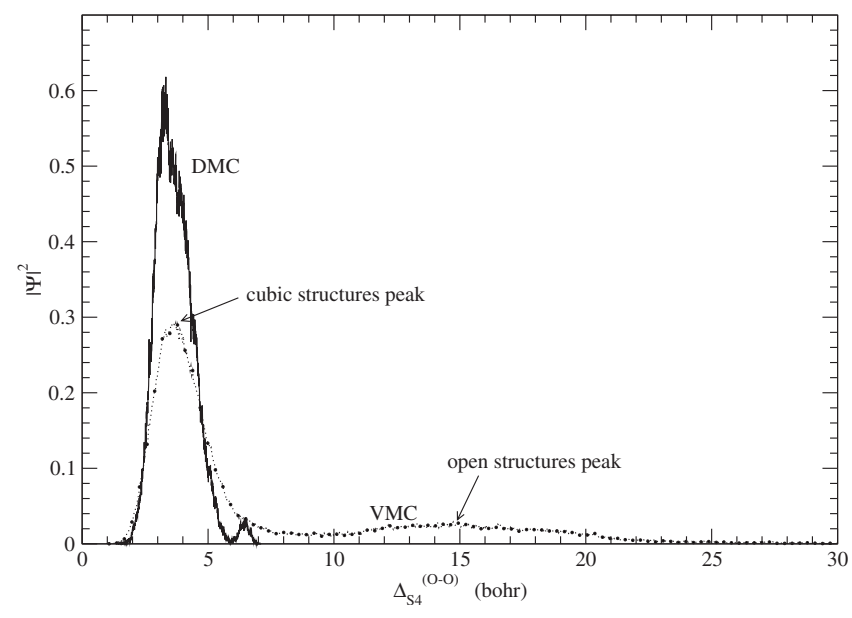

FIG. 5. A graph of the ground state density projected along the O-atoms SCA distance from the $D_{2 d}$ cube [cf. Eq. (24)] obtained from the VMC simulations (dots) and the GF-SPDMC simulation (lines).

higher energy states, including higher energy minima, obtained with the trial wave function in Eq. (17) is consistent with previous tests ${ }^{72}$ carried out with the same model.

\section{Structural analysis of the DMC ground state}

It is very instructive to compare the distributions from the variational simulation with the equivalent ones obtained from the GF-SPDMC walk. These are represented by black lines in Figs. 3-5. To generate $\Delta_{S 4}, \Delta_{D 2 d}$, and $\Delta_{S 4}^{(\mathrm{O}-0)}$ distributions from the GF-SPDMC walks, we write all the walking configurations at the end of ten independent 2000 step simulations to files. A total of 795863 configurations are generated in this manner and analyzed with the SCA. The histograms for the values of $\Delta_{S 4}, \Delta_{D 2 d}$, and $\Delta_{S 4}^{(\mathrm{O}-\mathrm{O})}$ are obtained by producing 1000 bins within the range. The distributions from the GF-SPDMC populations are all sharper than the corresponding ones obtained from the VMC walk. The $\Delta_{S 4}$ distribution from GF-SPDMC in Fig. 3 is a slightly asymmetric peak between 16 and $27 \mathrm{bohr}$, centered at 20 bohr. The $\Delta_{D 2 d}$ distribution from GF-SPDMC in Fig. 4 is between 10 and 20 bohr and peaks around 14 bohr. Finally, the $\Delta_{S 4}^{(\mathrm{O}-\mathrm{O})}$ from GF-SPDMC in Fig. 5 is between 2 and 5 bohr and peaks around 3 bohr.

A clear picture of the ground state wave function emerges by inspecting the graphs in Fig. 2, and the distributions obtained from the GF-SPDMC simulations in Figs. $3-5$. In particular, the relatively narrow $\Delta_{S 4}^{(\mathrm{O}-\mathrm{O})}$ distribution in Fig. 5, contained, for the most part, below 5 bohr tells us that the "shape" of the octamer in the ground state is cubic. The structure of the ground state of the water octamer, from the orientation degrees of freedom of the waters prospective, is more "fluidlike" relative to the translations. To be certain of the structural character of the guided DMC walk, a sample of 795863 configurations from the GF-SPDMC simulation are quenched as well. $100 \%$ of the replicas sampled from the GF-SPDMC walk collect in the $D_{2 d}$ minimum. The data in Figs. 3-5 and in Sec. III C confirm that $\left|\psi_{T}\right|^{2}$ has a substantial magnitude over the $S_{4}$ isomer, whereas the SCA distributions for the GF-SPDMC simulation and the result of the 795863 quenches confirm that the product $\psi_{0} \psi_{T}$ is exclusively in the 
$D_{2 d}$ well. Therefore, we conclude with confidence that $\psi_{0}$ must have no significant magnitude over the $S_{4}$ cube, or any other minimum.

\section{CONCLUSIONS}

In the present article we report results of several theoretical investigations carried out on the TIP4P water octamer. We use the reweighted random series path integral, together with the stereographic projection manifolds to sample the orientations of the rigid moieties of the cluster. The stereographic projection coordinates allow the MC path integral simulations of aggregates of rigid tops without neglecting precession effects, which contribute a truncation error of $O\left(k_{m}^{-2}\right)$. The reweighted random series approach we use in the present work is cubically convergent. It is instructive to compare the GF-SPDMC ground state energy with the quantum internal energy at low temperatures for the TIP4P octamer. If converging properly, and at a sufficiently low temperature, the internal energy should approach the ground state energy. The agreement between $\langle E\rangle_{k_{m}=32, T=40 \mathrm{~K}}$ and $\langle E\rangle_{\text {GF-SPDMC }}$ is reassuring. The agreement we observe is a clear demonstration that the SPPI approach, with a reasonable amount of resources, is capable to simulate a complex system like the water octamer in the full temperature range, between the classical limit and at temperatures where the thermodynamics are dominated by the ground state. However, the $k_{m}=32$ energy in the $40-70 \mathrm{~K}$ range does have a finite, albeit small slope. Below $30 \mathrm{~K}$ the energy drops toward the classical energy rather abruptly, indicating that its estimator is not converging uniformly in the 10-30 K range. On the full scale, the onset of an energy plateau at low temperatures is visible in Fig. 1(a). Since, our choice of $40 \mathrm{~K}$ is completely arbitrary, estimating the ground state energy from path integral simulations in this manner should only be good to one figure at best.

We can compare the thermodynamic properties of the water octamer obtained with the TIP4P potential in the present study, with those obtained from the SPC potential in Ref. 67. We have a number of minima of the SPC octamer from previous work, ${ }^{67}$ and using them we produce graphs of the two measures $\Delta_{D 2 d}$ and $\Delta_{D 2 d}^{(\mathrm{O}-\mathrm{O})}$ in Fig. 2, since the global minimum for the SCP isomer is the $D_{2 d}$ cube, and the next isomer in energy is the $S_{4}$ cube. The all-atoms SCA distance from their respective global minima is compared with the same measure used for the TIP4P octamer in Fig. 6. We use black squares for the TIP4P surface and white squares for the SPC surface. The energy of the global minimum $\left(D_{2 d}\right.$ cube) is 0.8 mhartree deeper for the SCP octamer. Beside the minor shifting in the absolute energy, the two sets of data in Fig. 6 differ in other details. All the cubic isomers of the SPC surface have a $\Delta_{D 2 d}^{(\mathrm{O}-\mathrm{O})}$ smaller than 5 bohr. The $D_{2 d}$ and the $S_{4}$ cubes are nearly equally close in energy, even though the role of global minimum between the two models is exchanged between the two hydrogen bonds arrangements. However, the energy gap between the global minimum and the group of noncubic structures is significantly smaller for the SPC model.

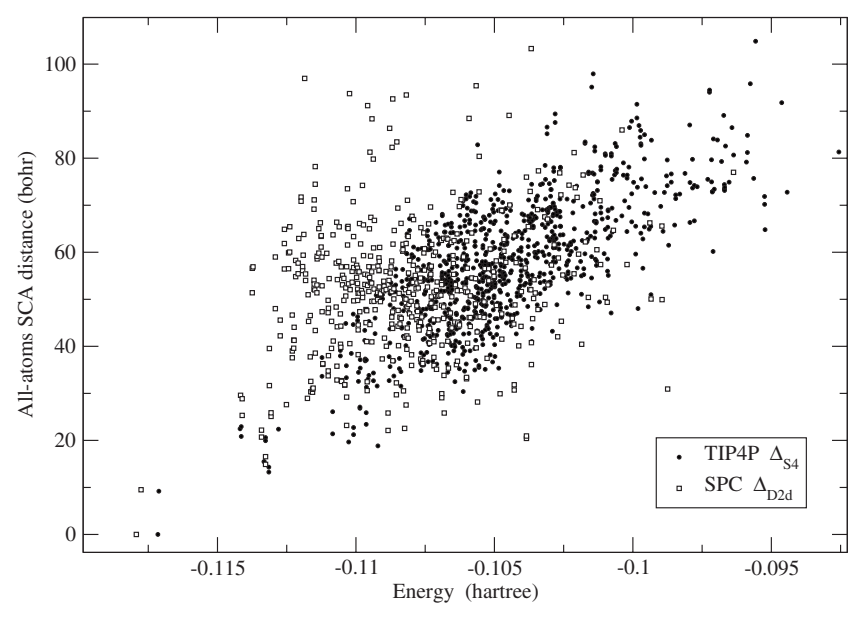

FIG. 6. A plot of the all-atoms SCA distance [cf. Eq. (22)] from the global minimum $\left(S_{4}\right.$ cube) as a function of energy measured for all distinct minima of the TIP4P water octamer (black squares), compared to the same measure from the global minimum $\left(D_{2 d}\right.$ cube) for the SCP octamer.

We compare the internal energy at finite temperature for the two potentials. The energy data for the SPC octamer used to compare with the TIP4P results were obtained during the work published in Ref. 67. The absolute values of the $k_{m}$ $=0$ and $k_{m}=32$ are $1-2$ mhartree deeper at $50 \mathrm{~K}$ for the SPC octamer. However, the gap between the classical and quantum internal energy at $50 \mathrm{~K}$ is statistically indistinguishable between the two models. In Ref. 67 we obtain a melting peak at $140 \mathrm{~K}$ in the classical heat capacity of the SCP octamer that is "washed away" almost entirely by the quantum effects. For the TIP4P octamer, by contrast, the classical melting peak at $210 \mathrm{~K}$ shifts to $160 \mathrm{~K}$ in the quantum limit. The fact that the energy gap between cubic and noncubic configurations is smaller for the SPC model provides an explanation for the observed differences in the classical and quantum heat capacities.

The structural analysis of the GF-SPDMC walk gives unequivocal evidence that the ground state wave function of the TIP4P octamer has $D_{2 d}$ symmetry, despite the large "fluidlike" behavior of the wave function along the orientation degrees of freedom relative to the translation ones. Given the fact that $\psi_{T}$, covers both the $D_{2 d}$, and the $S_{4}$ wells, as the distributions in Figs. 3 and 4 indicate, and the quenching of a large number of structures confirms that the distributions of $\psi_{0} \psi_{T}$, is physically relevant and provides important information. Since the distributions for $\psi_{0} \psi_{T}$ do not contain, as far as we can tell, any minimum other than the $D_{2 d}$ cube, we conclude with confidence that the ground state wave function is localized within the $D_{2 d}$ cube and other minima occur in the ground state with a probability less than one part in 795863 . We repeat all the SPDMC simulations for the SPC octamer and we find that its ground state wave function is predominantly within the $D_{2 d}$ well. One of the motivations for the present work is to determine if the characterization of the ground state wave function of the water octamer could offer a glimpse into the role that quantum effects play in determining important simulated properties. It does seem possible that the smearing of the hydrogen atoms by quantum fluctuations in the ground state is sufficiently extensive to wash out 
largely any differences predicted by slightly different models of the potential energy surfaces. We find that, despite the profound structural differences among the two global minima, the zero point energy differences are small, and despite the broad distribution of orientations of the hydrogen bonds, the ground state wave function is largely contained inside either a single basin of attraction with the most symmetric structure. In that sense, the smearing of the hydrogen atoms by quantum fluctuations in the ground state, and by thermal effects below melting, washes out some of the differences in the models.

Which potential energy model best represents water clusters remains an open and difficult question. However, the results we present suggest that properties, which depend on relative energy values of the clusters as a function of size at or near the ground state, should be very similar for both models in the classical and quantum limits. Clearly, a number of additional studies are warranted to generalize our findings beyond the SCP and TIP4P octamer cases, especially if information like the binding energy of hydrogen with water clusters is desired. For example, it may be insightful to compare our results with simulations obtained with models that may produce ground state wave functions contained inside a single basin of attraction other than the $D_{2 d}$ cube, such as the empirical modified potential (EMP), ${ }^{13}$ which could favor the $S_{4}$ isomer for the ground state.

Finally, the comparisons between the VMC and GFSPDMC distributions in Figs. 3-6 give us valuable information about the level of accuracy of the variational scheme used in the present investigation. The "thermal" trial wave function in Eq. (17) samples configurations that are liquidlike for both the orientations and the translations of the waters. The oversampling of high energy states observed in this work is consistent with the observations made in Ref. 72 when the same type of wave function is tested on the quartic double well and the Stockmayer trimer. Clearly, the trial wave function requires an optimal parameter $\alpha$, which corresponds to a thermal state with a significant amount of melting of the molecular framework. This type of oversampling along the translational degrees of freedom could be curbed by alternative trial functions, such as e.g.,

$$
\psi_{T}(q)=A \exp \left\{-\frac{1}{2} \alpha V_{\mathrm{eff}}(q)\right\}
$$

where

$$
V_{\mathrm{eff}}(q)=\frac{g_{\mu \nu}\left(q_{0}\right)\left(q^{\mu}-q_{0}^{\mu}\right)\left(q^{\nu}-q_{0}^{\nu}\right)}{(\Delta \tau)^{2}}+V
$$

In Eq. (35), $g_{\mu \nu}\left(q_{0}\right)$ is the Hessian metric tensor evaluated at $q_{0}$, and the latter is a sensible reference configuration, such as the global minimum. The parameter $\Delta \tau$, in units of time, can be optimized starting with a large value since as $\Delta \tau$ $\rightarrow \infty$, one obtains the trial wave function in Eq. (17). A more precise guiding function like in Eqs. (34) and (35) is desirable to reduce the statistical error of guided SPDMC. However, there is a real danger of biasing the DMC walk into a single basin of attraction (which could also be the incorrect one), if Eqs. (34) and (35) are used without any previous knowledge of the system. The double sum on the right hand side of Eq. (35) does not have to include all the degrees of freedom. It may be advisable to only use those degrees of freedom which are not broadly distributed, such as the positions of the centers as in the present case. Finally, the optimization of the parameter $\alpha$ can be carried out using parallel tempering. We did not engage in a systematic investigation of the behavior of the trial function in Eqs. (34) and (35) because the issue is tangential to the focus of the present work. However, a careful systematic investigation of such variational model is clearly warranted and should be carried out in simpler Euclidean manifolds first, ${ }^{91-102}$ using mixed atomic clusters and a wide range of mass values.

\section{ACKNOWLEDGMENTS}

We thank Professor K. D. Jordan for suggesting that we examine the quantum melting behavior of the water octamer using the TIP4P potential. This work has been supported by the National Science Foundation (Grant No. CHE0554922). Additionally, E.C. acknowledges the donors of the Petroleum Research Fund, administered by the ACS (Grant No. 48146B6), The Stacy Ann Vitetta '82 Professorship Fund, and The Ellington Beavers Fund for Intellectual Inquiry from Arcadia University for partial support of this research.

${ }^{1}$ J. C. Owicki, L. L. Shipman, and H. A. Scheraga, J. Phys. Chem. 79, 1794 (1975)

${ }^{2}$ H. Matsuoka, E. Clementi, and M. Yoshimine, J. Chem. Phys. 64, 1351 (1976).

${ }^{3}$ F. H. Stillinger and C. W. David, J. Chem. Phys. 73, 3384 (1980).

${ }^{4}$ G. Brink and L. Glasser, J. Phys. Chem. 88, 3412 (1984).

${ }^{5}$ D. Belford and E. S. Campbell, J. Chem. Phys. 86, 7013 (1987).

${ }^{6}$ C. E. Dykstra, J. Chem. Phys. 91, 6472 (1989).

${ }^{7}$ R. J. Wawak, M. M. Wimmer, and H. A. Scheraga, J. Phys. Chem. 96, 5138 (1992).

${ }^{8}$ D. J. Wales and I. Ohmine, J. Chem. Phys. 98, 7245 (1993).

${ }^{9}$ C. J. Tsai and K. D. Jordan, Chem. Phys. Lett. 213, 181 (1993).

${ }^{10}$ C. J. Tsai and K. D. Jordan, J. Phys. Chem. 97, 5208 (1993).

${ }^{11}$ K. N. Kirschner and G. C. Shields, Int. J. Quantum Chem. 52, 349 (1994)

${ }^{12}$ J. M. Pedulla, F. Vila, and K. D. Jordan, J. Chem. Phys. 105, 11091 (1996).

${ }^{13}$ U. Buck, I. Ettischer, M. Melzer, V. Buch, and J. Sadlej, Phys. Rev. Lett. 80, 2578 (1998).

${ }^{14}$ J. M. Pedulla, K. Kim, and K. D. Jordan, Chem. Phys. Lett. 291, 78 (1998).

${ }^{15}$ I. M. B. Nielsen, E. T. Seidl, and C. L. Janssen, J. Chem. Phys. 110, 9435 (1999).

${ }^{16}$ J. Qian, E. Stöckelmann, and R. Hentschke, J. Mol. Model. 5, 281 (1999).

${ }^{17}$ B. Hartke, Z. Phys. Chem. 214, 1251 (2000).

${ }^{18}$ F. F. Guimarães, J. Belchior, R. L. Johnston, and C. Roberts, J. Chem. Phys. 116, 8327 (2002).

${ }^{19}$ H. Kabrede and R. Hentschke, J. Phys. Chem. B 107, 3914 (2003).

${ }^{20}$ J. K. Kazimirski and V. Buch, J. Phys. Chem. A 107, 9762 (2003).

${ }^{21}$ J. Cui, H. Liu, and K. D. Jordan, J. Phys. Chem. 110, 18872 (2006).

${ }^{22}$ A. DeFusco, D. Schofield, and K. D. Jordan, Mol. Phys. 105, 2681 (2007).

${ }^{23}$ C. J. Tsai and K. D. Jordan, J. Chem. Phys. 95, 6472 (1991).

${ }^{24}$ C. J. Tsai and K. D. Jordan, J. Chem. Phys. 99, 6957 (1993).

${ }^{25}$ L. X. Dang and T.-M. Chang, J. Chem. Phys. 106, 8149 (1997).

${ }^{26}$ J. M. Pedulla and K. D. Jordan, Chem. Phys. 239, 593 (1998).

${ }^{27}$ D. Laria, J. Rodriguez, C. Dellago, and D. Chandler, J. Phys. Chem. A 105, 2646 (2001)

${ }^{28}$ P. A. Nigra, M. C. Cargnano, and S. Kais, J. Chem. Phys. 115, 2621 (2001).

${ }^{29}$ M. Ocasio and G. López, Chem. Phys. Lett. 356, 168 (2002).

${ }^{30}$ F. Wang and K. D. Jordan, J. Chem. Phys. 119, 11645 (2003).

${ }^{31}$ A. Tharrington and K. D. Jordan, J. Phys. Chem. A 107, 7380 (2003). 
${ }^{32}$ S. S. Xantheas and E. Aprà, J. Chem. Phys. 120, 823 (2004).

${ }^{33}$ E. Johansson, K. Bolton, and P. Ahlström, J. Chem. Phys. 123, 024504 (2005).

${ }^{34}$ Y. C. Choi, C. Pak, and K. S. Kim, J. Chem. Phys. 124, 094308 (2006).

${ }^{35}$ M. N. Slipchenko, K. E. Kuyanov, B. G. Sartakov, and A. F. Vilesov, J. Chem. Phys. 124, 241101 (2006).

${ }^{36}$ L. Turi, Á. Madarász, and P. J. Rossky, J. Chem. Phys. 125, 014308 (2006).

${ }^{37}$ A. Krishtal, P. Senet, M. Yang, and C. Van Alsenoy, J. Chem. Phys. 125, 034312 (2006).

${ }^{38}$ H. Tachikawa, J. Chem. Phys. 125, 144307 (2006).

${ }^{39}$ C. Caleman and D. van der Spoel, J. Chem. Phys. 125, 154508 (2006).

${ }^{40}$ I. B. Müller and L. S. Cederbaum, J. Chem. Phys. 125, 204305 (2006).

${ }^{41}$ J. Hernández-Rojas, B. S. González, T. James, and D. J. Wales, J. Chem. Phys. 125, 224302 (2006).

${ }^{42}$ T. James, D. J. Wales, and J. H. Rojas, J. Chem. Phys. 126, 054506 (2007).

${ }^{43}$ P. A. Frantsuzov and V. A. Mandelshtam, J. Chem. Phys. 128, 094304 (2008).

${ }^{44}$ J. Zielkiewicz, J. Chem. Phys. 123, 104501 (2005).

${ }^{45}$ D. J. Price and C. L. Brooks III, J. Chem. Phys. 121, 10096 (2004).

${ }^{46}$ H. Yu, T. Hansson, and W. F. van Gunsteren, J. Chem. Phys. 118, 221 (2003).

${ }^{47}$ M. Předota, A. A. Chialvo, and P. T. Cummings, Fluid Phase Equilib. 183-184, 295 (2001).

${ }^{48}$ M. W. Mahoney and W. L. Jorgensen, J. Chem. Phys. 115, 10758 (2001).

${ }^{49}$ J. Lobaugh and G. A. Voth, J. Chem. Phys. 106, 2400 (1997).

${ }^{50}$ W. L. Jorgensen, J. Chandrasekhar, J. D. Madura, R. W. Impey, and M. L. Klein, J. Chem. Phys. 79, 926 (1983).

${ }^{51}$ M. Shiga and W. Shinoda, J. Chem. Phys. 123, 134502 (2005).

${ }^{52}$ C. J. Burnham, J. Li, S. S. Xantheas, and M. Leslie, J. Chem. Phys. 110, 4566 (1999).

${ }^{53}$ J. L. F. Abascal, E. Sanz, R. G. Fernández, and C. Vega, J. Chem. Phys. 123, 234505 (2005)

${ }^{54}$ S. Alavi, J. A. Ripmeester, and D. D. Klug, J. Chem. Phys. 123, 024507 (2005).

${ }^{55}$ S. Alavi, J. A. Ripmeester, and D. D. Klug, J. Chem. Phys. 123, 051107 (2005).

${ }^{56}$ S. Alavi, J. A. Ripmeester, and D. D. Klug, J. Chem. Phys. 124, 204707 (2006).

${ }^{57}$ G. Kerenskaya, I. U. Goldschleger, V. A. Apkarian, and K. C. Janda, J. Phys. Chem. A 110, 13792 (2006).

${ }^{58}$ S. Lee, P. Yedlapalli, and J. W. Lee, J. Phys. Chem. B 110, 26122 (2006).

${ }^{59}$ V. V. Struzhkin, B. Militzer, W. L. Mao, H.-K. Mao, and R. J. Hemley, Chem. Rev. 107, 4133 (2007).

${ }^{60}$ F. Sebastianelli, M. Xu, D. K. Kanan, and Z. Bacić, J. Phys. Chem. A. 111, 6115 (2007).

${ }^{61}$ S. Alavi, J. A. Ripmeester, and D. D. Klug, J. Chem. Phys. 126, 124708 (2007).

${ }^{62}$ L. Senadheera and M. S. Conradi, J. Phys. Chem. B 111, 12097 (2007).

${ }^{63}$ M. F. Russo, Jr. and E. Curotto, J. Chem. Phys. 118, 6806 (2003).

${ }^{64}$ M. F. Russo, Jr. and E. Curotto, J. Chem. Phys. 120, 2110 (2004).

${ }^{65}$ M. W. Avilés and E. Curotto, J. Chem. Phys. 122, 164109 (2005).

${ }^{66}$ E. Curotto, J. Chem. Phys. 123, 134102 (2005).

${ }^{67}$ S. F. Langley, E. Curotto, D. L. Freeman, and J. D. Doll, J. Chem. Phys. 126, 084506 (2007).

${ }^{68}$ M. W. Avilés, M. L. McCandless, and E. Curotto, J. Chem. Phys. 128, 124517 (2008).

${ }^{69}$ M. W. Avilés, P. T. Gray, and E. Curotto, J. Chem. Phys. 124, 174305 (2006).

${ }^{70}$ E. Curotto, D. L. Freeman, and J. D. Doll, J. Chem. Phys. 128, 204107 (2008).

${ }^{71}$ M. W. Avilés and E. Curotto, J. Phys. Chem. A 111, 2610 (2007).

${ }^{72}$ T. Luan, E. Curotto, and M. Mella, J. Chem. Phys. 128, 164102 (2008).

${ }^{73}$ M. Mella and D. C. Clary, J. Chem. Phys. 119, 10048 (2003).

${ }^{74}$ M. Mella, D. C. Clary, J.-L. Kuo, and M. L. Klein, Phys. Chem. Chem. Phys. 7, 2324 (2005)

${ }^{75}$ R. A. Kuharski and P. J. Rossky, J. Chem. Phys. 82, 5164 (1985).

${ }^{76}$ D. Marx and M. H. Müser, J. Phys.: Condens. Matter 11, R117 (1999).

${ }^{77}$ D. Marx and P. Nielaba, Phys. Rev. A 45, 8968 (1992).

${ }^{78}$ D. C. Clary, J. Chem. Phys. 116, 9892 (2002).

${ }^{79}$ V. Buch, J. Chem. Phys. 97, 726 (1992).
${ }^{80}$ A. Sarsa, K. E. Schmidt, and J. W. Moskowitz, J. Chem. Phys. 113, 44 (2000).

${ }^{81}$ D. C. Clary, J. Chem. Phys. 114, 9725 (2001).

${ }^{82}$ H. Jiang, M. Z. Xu, J. M. Hutson, and Z. Bacìc, J. Chem. Phys. 123, 054305 (2005).

${ }^{83}$ H. Jiang and Z. Bacìc, J. Chem. Phys. 122, 244306 (2005).

${ }^{84}$ F. Paesani, F. A. Gianturco, and K. B. Whaley, J. Chem. Phys. 115, 10225 (2001).

${ }^{85}$ J. W. Moskowitz, Z. Bacìc, A. Sarsa, and K. E. Schmidt, J. Chem. Phys. 114, 10294 (2001).

${ }^{86}$ V. Melik-Alaverdian, N. E. Bonesteel, and G. Ortiz, Phys. Rev. Lett. 79, 5286 (1997).

${ }^{87}$ M. H. Kalos, D. Levesque, and L. Verlet, Phys. Rev. A 9, 2178 (1974).

${ }^{88}$ M. H. Kalos and P. A. Whitlock, Monte Carlo Methods (Wiley, New York, 1986)

${ }^{89}$ J. B. Anderson, J. Chem. Phys. 63, 1499 (1975).

${ }^{90}$ D. M. Ceperley and D. Alder, Science 231, 555 (1986).

${ }^{91}$ S. W. Rick and J. D. Doll, Chem. Phys. Lett. 188, 149 (1992).

${ }^{92}$ S. W. Rick, D. L. Lynch, and J. D. Doll, J. Chem. Phys. 95, 3506 (1991).

${ }^{93}$ R. Guardiola and J. Navarro, J. Chem. Phys. 128, 144303 (2008).

${ }^{94}$ E. Coccia, F. Marinetti, E. Bodo, and F. A. Gianturco, J. Chem. Phys. 128, 134511 (2008).

${ }^{95}$ R. Springall, M. C. Per, S. P. Russo, and I. K. Snook, J. Chem. Phys. 128, 114308 (2008).

${ }^{96}$ P. H. Acioli, Z. Xie, B. J. Braams, and J. M. Bowman, J. Chem. Phys. 128, 104318 (2008).

${ }^{97}$ I. Beslić, L. V. Markić, and J. Boronat, J. Chem. Phys. 128, 064302 (2008).

${ }^{98}$ A. Viel, K. B. Whaley, and R. J. Wheatley, J. Chem. Phys. 127, 194303 (2007).

${ }^{99}$ O. Vendrell, F. Gatti, D. Lauvergnat, and H.-D. Meyer, J. Chem. Phys. 127, 184302 (2007).

${ }^{100}$ R. Guardiola, O. Kornilov, J. Navarro, and J. P. Toennies, J. Chem. Phys. 124, 084307 (2006).

${ }^{101}$ M. P. Nightingale and P.-N. Roy, J. Phys. Chem. A 110, 5391 (2006).

${ }^{102}$ M. Mella, G. Calderoni, and F. Cargnoni, J. Chem. Phys. 123, 054328 (2005).

${ }^{103}$ R. Hołyst, D. Plewczyński, A. Aksimentiev, and K. Burdzy, Phys. Rev. E 60, 302 (1999).

${ }^{104}$ C. Predescu, e-print arXiv:cond-mat/0302171.

${ }^{105}$ C. Predescu, D. Sabo, and J. D. Doll, J. Chem. Phys. 119, 4641 (2003).

${ }^{106}$ C. Predescu and J. D. Doll, J. Chem. Phys. 117, 7448 (2002).

${ }^{107}$ C. Predescu, D. Sabo, J. D. Doll, and D. L. Freeman, J. Chem. Phys. 119, 10475 (2003).

${ }^{108}$ C. Predescu, D. Sabo, J. D. Doll, and D. L. Freeman, J. Chem. Phys. 119, 12119 (2003).

${ }^{109}$ D. Sabo, C. Predescu, and J. D. Doll, J. Chem. Phys. 121, 856 (2004).

${ }^{110}$ C. Predescu, P. A. Frantsuzov, and V. A. Mandelshtam, J. Chem. Phys. 122, 154305 (2005).

${ }^{111}$ S. W. Rick and A. D. J. Haymet, J. Chem. Phys. 118, 9291 (2003).

${ }^{112}$ B. A. Berg and W. Yang, J. Chem. Phys. 127, 224502 (2007).

${ }^{113}$ N. Metropolis, A. W. Rosenbluth, M. N. Rosenbluth, A. M. Teller, and E. Teller, J. Chem. Phys. 21, 1087 (1953).

${ }^{114}$ M. P. Allen and D. J. Tildesley, Computer Simulations of Liquids (Clarendon, Oxford, 1987).

${ }^{115}$ D. Frenkel and B. Smit, Understanding Molecular Simulations, 2nd ed. (Academic, New York, 1996).

${ }^{116}$ W. D. Curtis and F. R. Miller, Differential Manifolds and Theoretical Physics (Academic, New York, 1985).

${ }^{117}$ H. Kleinert, Path integrals in Quantum Mechanics, Statistics and Polymer Physics (World Scientific, Singapore, 1990).

${ }^{118}$ J. K. Lee, J. A. Barker, and F. F. Abraham, J. Chem. Phys. 58, 3166 (1973).

${ }^{119}$ M. Falcioni and M. W. Deem, J. Chem. Phys. 110, 1754 (1999).

${ }^{120}$ J. P. Neirotti, F. Calvo, D. L. Freeman, and J. D. Doll, J. Chem. Phys. 112, 10340 (2000).

${ }^{121}$ F. Calvo, J. P. Neirotti, D. L. Freeman, and J. D. Doll, J. Chem. Phys. 112, 10350 (2000).

${ }^{122}$ J. P. Neirotti, D. L. Freeman, and J. D. Doll, Phys. Rev. E 62, 7445 (2000).

${ }^{123}$ E. Curotto, A. Matro, D. L. Freeman, and J. D. Doll, J. Chem. Phys. 108, 729 (1998). 
The Journal of Chemical Physics is copyrighted by the American Institute of Physics (AIP). Redistribution of journal material is subject to the AIP online journal license and/or AIP copyright. For more information, see http://ojps.aip.org/jcpo/jcper/jsp 